\title{
Signo del aire creciente
}

\section{Air crescent sign}

Ana Cristina Manzano, MD. (1), Pablo Ramón Gil TorRes, MD. ${ }^{(2)}$, Carlos Andrés Celis Preciado, MD. ${ }^{(3)}$

${ }^{(1)}$ Radióloga, Departamento de Radiología, Hospital Universitario San Ignacio. Bogotá, Colombia.

(2)Residente de Medicina Interna, Fundación Valle del Lili, Universidad ICESI. Cali, Colombia.

${ }^{(3)}$ Internista, Neumólogo, Unidad de Neumología, Hospital Universitario San Ignacio. Pontificia Universidad Javeriana. Bogotá, Colombia.

Correspondencia: Ana Cristina Manzano, correo electrónico: crispetamanzano@gmail. com

Recibido: 29/11/13, Aprobado: 20/02/14.
La Sociedad Fleischner define en su Glosario de Términos de Radiología Torácica a este hallazgo como una colección de aire en forma de luna creciente que separa la pared de una cavidad, de una masa interna, que puede observarse en la radiografía y la tomografía de tórax (1). La forma de dicha colección puede variar desde forma de medialuna hasta encapsular totalmente la lesión. El signo del aire creciente también se ha descrito como "signo del menisco pulmonar" o "signo de la media luna" (2).

La causa más común de este signo es la aspergilosis angioinvasiva en la cual las hifas del hongo invaden las paredes de los vasos sanguíneos pulmonares generando hemorragia, trombosis arterial y finalmente infarto pulmonar, el cual se presenta como una zona de consolidación. Con el tiempo el centro infartado se retrae y es reabsorbido por los leucocitos formando un área de tejido desvitalizado que se desprende del resto de la consolidación y "cae" a la región dependiente del infarto. El aire localizado entre el tejido desvitalizado y el parénquima consolidado que lo rodea, resulta en la forma de media luna o del aire creciente. La formación de este signo es tardía y depende de la función de los neutrófilos, de

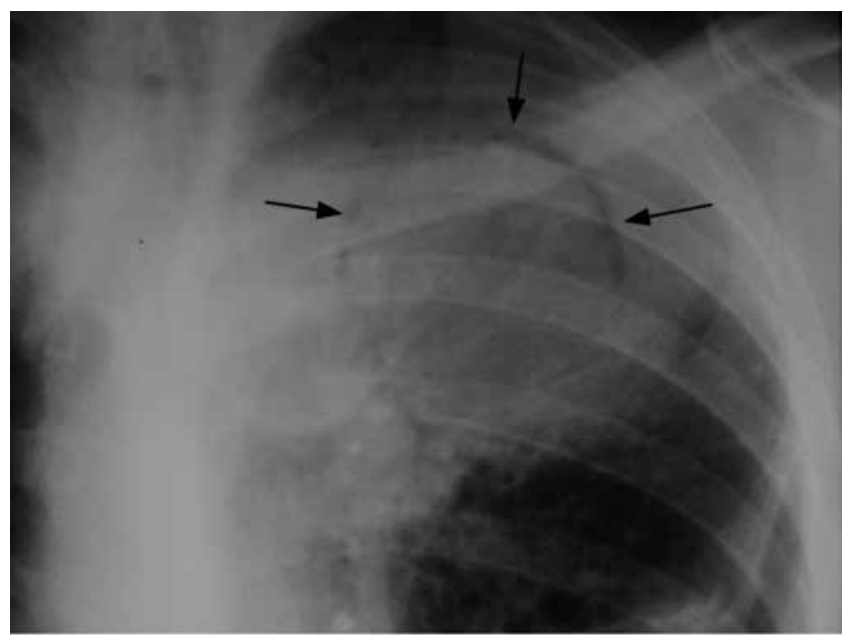

Figura 1. Signo del aire creciente. Obsérvese(flechas) el aire en forma de cresciente, que define una zona de desprendiento de parénquima infartado ,en un paciente con Aspergilosis angioinvasiva 
ahí que generalmente se observe en pacientes inmunosuprimidos (p. ej. en quimioterapia) durante el período de recuperación celular (3). Es especialmente frecuente en pacientes en quimioterapia para linfoma o leucemia.

Algunos autores hacen énfasis en diferenciarlo del signo de Monad del micetoma (3). En este último una bola de hongos, la cual está formada por hifas condensadas enmarañadas junto con moco, fibrina y restos celulares, es la que invade un espacio creado por una enfermedad fibrocavitaria previa (p. ej. tuberculosis o sarcoidosis). A diferencia del signo del aire creciente, la apariencia radiográfica es la de una masa que cambia con la posición del paciente dentro de una cavidad preexistente.

El signo del aire creciente también ha sido descrito en infección por otros hongos (4), quiste hidatídico (5), neumonía estafilocócica (6), infección por Nocardia, aneurisma de Rasmussen en una cavidad tuberculosa (2), hematoma (7), gangrena (8), carcinoma escamocelular (9) y adenocarcinoma (10).

\section{Bibliografía}

1. Fleishnner Society: Glossary of terms for thoracic imaging. Radiology. 2008;46: 697-722.

2. Fred HL, Gardiner CL. The air crescent sign: causes and characteristics. Tex Heart Inst J. 2009;36:264-5.

3. Abramson S. The air crescent sign. Radiology. 2001;218:230-2.

4. Tonelli AR, Khalife WT, Cao M, Young VB. Spherules, hyphae, and air-crescent sign. Am J Med Sci 2008; 335: 504-6.

5. Zidi A, Ben Miled-Mrad K, Hantous-Zannad S, Fathallah B, Mestiri I, Baccouche I, Djilani H. Computed tomography of complicated pulmonary hydatid cyst by rupture in the bronchi. J Radiol. 2007;88:59-64.

6. Gold W, Vellend H, Brunton J. The air crescent sign caused by Staphylococcus aureus lung infection in a neutropenic patient with leukemia. Ann Intern Med. 1992; 116: 910-1.

7. Bard R, Hassani N. Crescent sign in pulmonary hematoma. Respiration. 1975;32:247-51.

8. Reich JM. Pulmonary gangrene and the air crescent sign. Thorax. 1993;48:70-4.

9. Lee KL, Liang HH, Chung CL, Hsiao SH, Shih CH. Pulmonary air crescent sign. JAMA Surg. 2014;149:97-8.

10. Wang LF, Chu H, Chen YM, Perng RP. Adenocarcinoma of the lung presenting as a mycetoma with an air crescent sign. Chest. 2007;131:1239-42. 